\title{
Sampling Nonuniformity in the SDC Calorimeter EM/HAD Boundary
}

\author{
Dan Green \\ Fermi National Accelerator Laboratory \\ P.O. Box 500, Batavia, Illinois 60510
}

April 1993 


\section{Disclaimer}

This report was prepared as an account of work sponsored by an agency of the United States Government. Neither the United States Government nor any agency thereof, nor any of their employees, makes any warranty, express or implied, or assumes any legal liability or responsibility for the accuracy, completeness, or usefulness of any information, apparatus, product, or process disclosed, or represents that its use would not infringe privately owned rights. Reference herein to any specific commercial product, process, or service by trade name, trademark, manufacturer, or otherwise, does not necessarily constitute or imply its endorsement, recommendation, or favoring by the United States Government or any agency thereof. The views and opinions of authors expressed herein do not necessarily state or reflect those of the United States Government or any agency thereof. 


\title{
SAMPLING NONUNIFORMITY IN THE SDC CALORIMETER EM/HAD BOUNDARY
}

\author{
Dan Green \\ Fermi National Accelerator Laboratory \\ Batavia, Illinois
}

April 1993

\section{Introduction}

The SDC steel hadronic (HAD) calorimeter absorber structure has been designed to be of low magnetic reluctance, high rigidity, and excellent hermiticity [1]. Transverse scintillator tiles staggered longitudinally allow one to assemble the hadronic calorimeter compartment with no transverse dead areas between tiles. The construction is illustrated in Fig. 1. Clearly, the structure affords many advantages, among them hermiticity. The only problem is that, at the boundary of the structure, as at the EM/HAD boundary, the steel sampling fraction for adjacent transverse towers is different. In principle then, the adjacent towers will have different energy calibrations, and different energy resolutions. The aim of this note is to examine the size of these effects and, if necessary, explore ways to minimize their impact.

\section{Leakage of EM Energy}

One effect of the different depth of HAD steel at the EM/HAD boundary is in the energy leakage from the EM compartment. Fiscal considerations argue to make the fine sampling EM compartment as thin as possible. However, high energy EM showers will then leak into the HAD compartment. The correction of the total energy for the leakage fluctuations will then depend, numerically, on the details of the EM/HAD boundary.

In order to look at this effect, test beam data using the "Hanging File" (HF) apparatus [2] was used. The highest energy electron data, $170 \mathrm{GeV}$ incident energy, was used. The HF absorber consisted of $40 \mathrm{~Pb}$ plates, each 1/8" thick, followed by 55 plates of 1 " Fe. Each layer was separately read out by a PMT. The data set had a fractonal energy error of $1.5 \%$, presumably due to the nonuniformities inherent in the construction and the fluctuations due to the sampling fraction.

Leakage was studied by artificially truncating the EM compartment energy sum to less than the full 40 tile sum. The resulting induced leakage energy error was unfolded in quadrature. The fractional energy error due to leakage as a function of the reduced EM compartment depth is shown in Fig. 2. For a depth $\sim 17$ radiation lengths (Xo) the leakage error is $\sim 2 \%$. This is outside the SDC specifications [1].

The energy for the stack truncated to $\sim 17$ Xo can be corrected by measuring the energy in the subsequent compartment, Ehad. Making event by event corrections, one can basically correct for the fluctuations in leakage energy caused by the conversion point fluctuations in the EM compartment. The correlation between Eem and Ehad is shown in Fig 3a. Clearly, 
the correlation allows us to restore the energy sum such that the leakage energy error is reduced to acceptable levels [1].

The SDC EM compartment is expected to be subjected to a large radiation field. The SDC design utilizes longitudinal segmentation to reduce the sensitivity of the EM energy measurement to radiation damage [3]. Either explicit E1/E2 segmentation or the "shower maximum" sample at fixed depth can be used [4]. In Fig. 3b is shown the correlation between the EM segmentation, E1em/E2em and the leakage energy, Ehad. Clearly, the two quantities are highly correlated. Basically they measure the same thing, the fluctuation in the conversion point. A large E2em/E1em indicates a late conversion, which, in turn, leads to a large leakage energy, Ehad. Therefore, it is expected that EM segmentation can be used to correct for leakage energy errors. Hence, these errors will not drive the design of the EM/HAD boundary.

\section{Hadronic Energy Measurement and the EM/HAD Boundary}

One expects that the measurement of hadronic energy will depend on the sampling fraction details near the EM/HAD boundary. Elementary considerations lead one to believe that a uniform sampling fraction leads to a minimum in the energy resolution. In order to study this problem, $\mathrm{HF}$ data with $270 \mathrm{GeV}$ incident pions was used. The energy from tiles behind the 1" plates near the EM/HAD boundary were dropped from the energy sum. In that way, "inert" plates could be "manufactured" so as to locally change the sampling fraction near the EM/HAD boundary. As shown in Fig. 1, this procedure is a rough approximation to the structure implied by the SDC design of the hadronic steel absorber [1].

Data were analyzed with $0,1,2$, and 3 "inert" plates. The resulting fractional energy error was measured, and the $5.5 \%$ energy error seen for uniform sampling was subtracted in quadrature. The resulting "induced" fractional energy error due to nonuniform sampling is shown in Fig. 4 as a function of the number of inert plates. The error is roughly linear in the number of inert plates, being $\sim 3 \% / 1$ " plate. The existence of 2 uncorrected plates would push the resolution outside the SDC specifications [1].

The nonuniform sampling will also cause a shift in the mean energy. The fractional shift in the mean energy as a function of the number of inert plates is shown in Fig. 5. The shift is roughly linear, consisting of $\sim 2.5 \% / 1$ " plate.

\section{Weighting Strategy}

The light output of a tile/fiber layer can be controlled by the thickness of the tile, the depth of the fiber insertion, the length of the fiber in the tile, "masking" of the assembly, and control of several other variables [1]. Therefore, it is legitimate to consider varying the light output of the tile directly behind the "inert" stack of plates which constitute the nonuniform sampling region. The "induced" fractional energy error due to the existence of one 1" inert Fe plate as a function of the "weight" = WT of the tile behind that plate is shown in Fig. 6. Clearly, the 3.8\% error seen in Fig. $4(\mathrm{WT}=1)$ can be substantially reduced at the "optimal weight" of WT $\sim 3$. Since Fig. 6 is the situation for double sampling at the EM/HAD boundary (2" of steel, followed by a weighted tile, followed by a stack of 1 " Fe plates and unweighted tiles), one might naively expect that a weight, WT = 2 would be optimal. Clearly, this is close to the exact situation. 
Data was analyzed as in Fig. 6 for 1,2, and 3 inert plates. The resulting induced fractional energy error and fractional shift in the mean energy at the "optimal weight" are plotted in Fig. 4 and Fig. 5, as the dotted lines, respectively. Clearly, the sensitivity of the fractional energy error to this specific nonuniformity in sampling can be reduced by a factor $\sim 2$. Within the errors of the small data sample used here, the "optimal weight" both minimizes the energy resolution and stabilizes the shift of the mean. As seen in Fig. 5, the optimally weighted EM/HAD boundary suffers a shift in the mean of $<2 \%$. Thus, the transversely adjacent towers will respond equally to $<2 \%$ in the mean, and with a resolution which suffers an additional contribution of $<5 \%$ for $<3$ " of "inert" plates at the EM/HAD boundary.

\section{Summary}

Mechanical construction may dictate sampling nonunifiormities in the SDC hadronic absorber. Leakage of EM energy does not inform on this problem if longitudinal sampling within the EM compartment is available. However, the sampling nonuniformity will cause an increase in the hadronic energy resolution and a shift in the energy mean. An independent weighting of the tile just downstream of the nonuniformity restores the hadronic response to within SDC specifications if the sampling at the EM/HAD boundary is $<3$ times the sampling of the bulk of the steel stack.

\section{References}

1. SDC Technical Design Report, SDC-92-101 (1992).

2. A. Beretvas et al., "Beam Tests of Composite Calorimeter Configurations from Reconfigurable-Stack Calorimeter", accepted by Nuc. Inst. Meth.

3. D. Green, A. Para and J. Hauptman, "Radiation Damage, Calibration and Depth Segmentation in Calorimeters", Fermilab-FN-565, May 1991.

4. A. Beretvas, D. Green, J. Marraffino and W. Wu, "Use of a Shower Maximum Detector to Reduce Radiation Damage Sensitivity in EM Calorimetry", Fermilab-FN600, December 1992. 


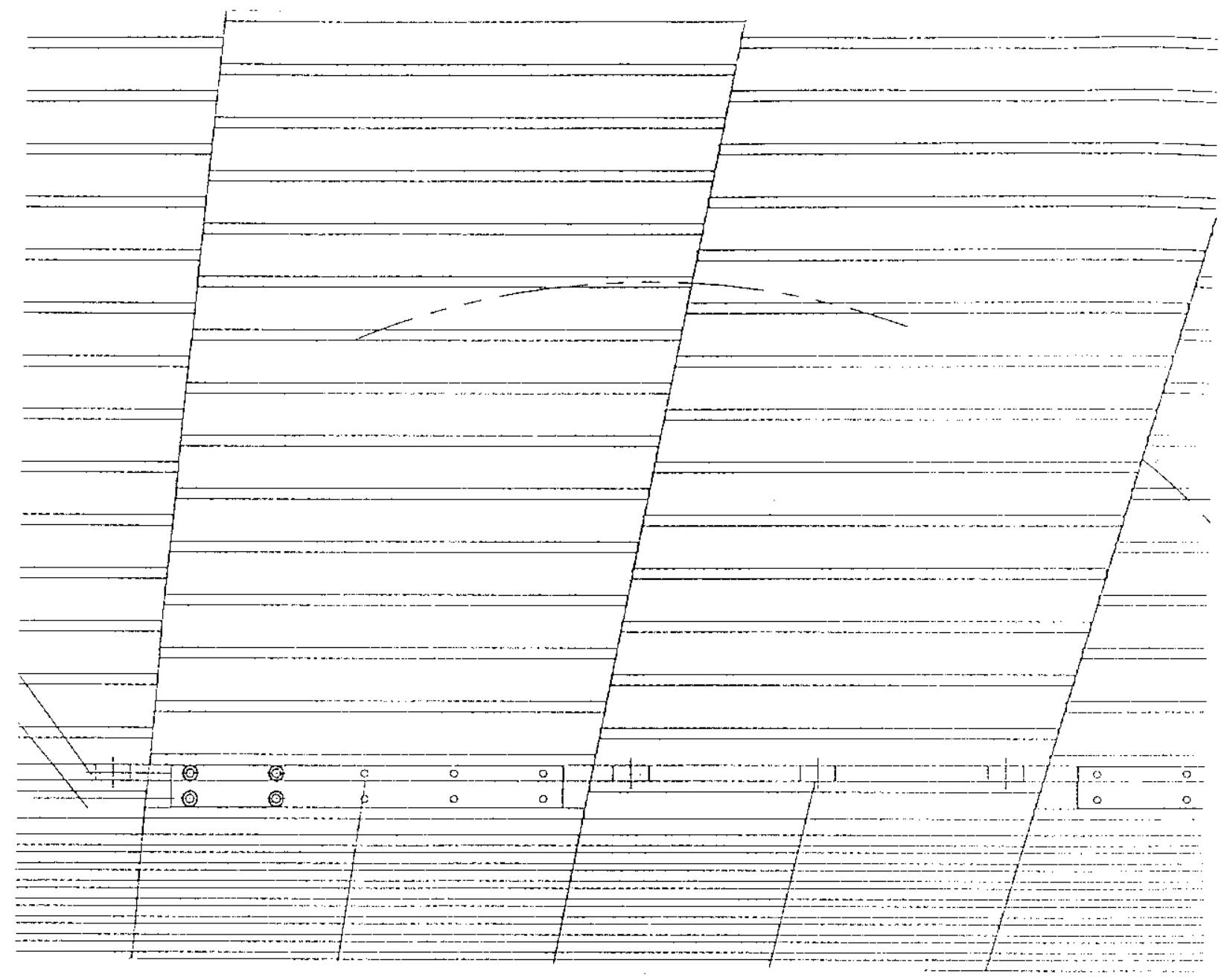

Figure 1 Schematic of the hadronic steel portion of the SDC barrel calorimeter. The construction method insures a low magnetic reluctance path for the solenoid flux return and a rigid hermetic structure. 


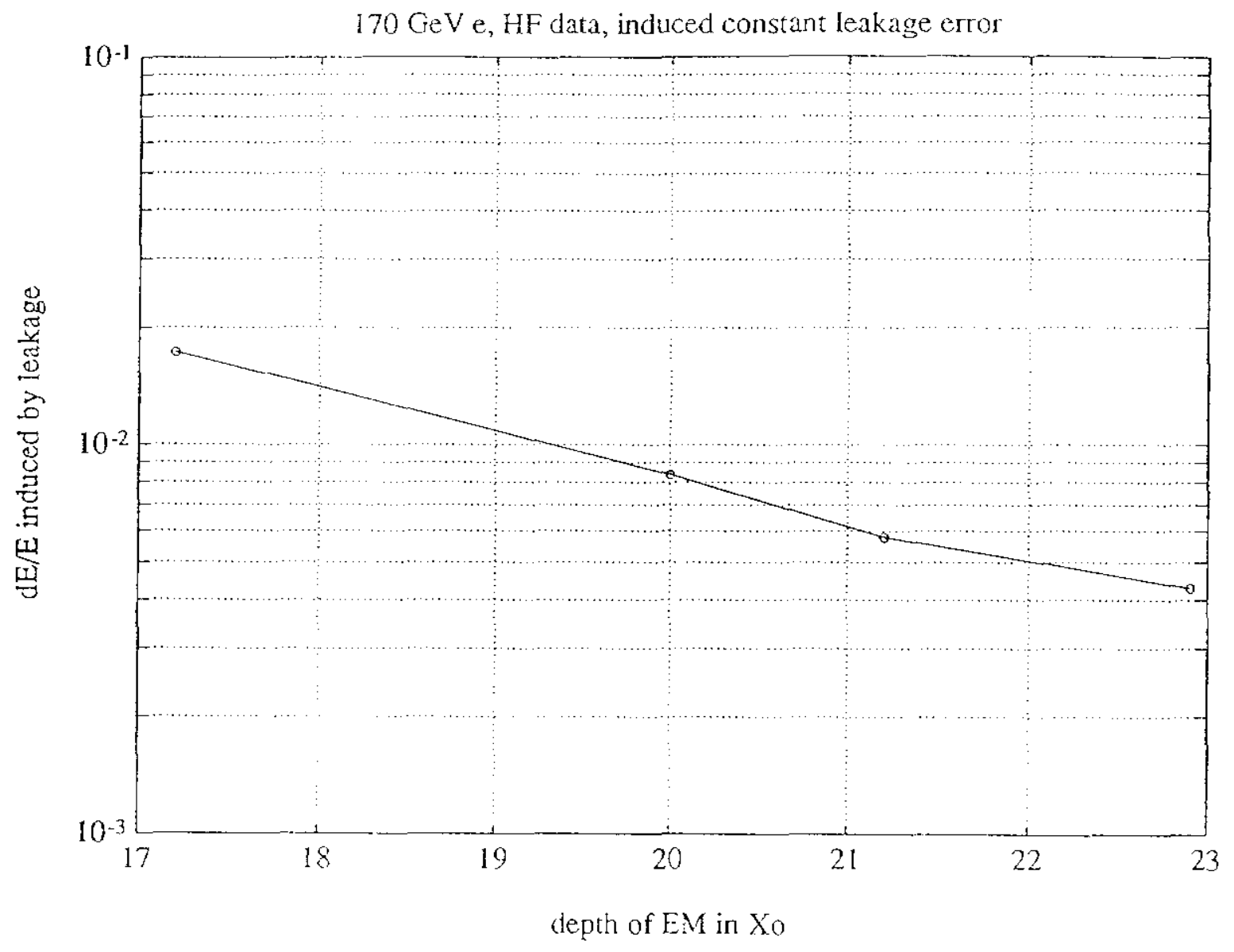

Figure 2 Hanging File (HF) data for $170 \mathrm{GeV}$ incident electrons. The fractional error caused by leakage fluctuations is shown as a function of the EM calorimeter depth in radiation length $(\mathrm{Xo})$ units. 

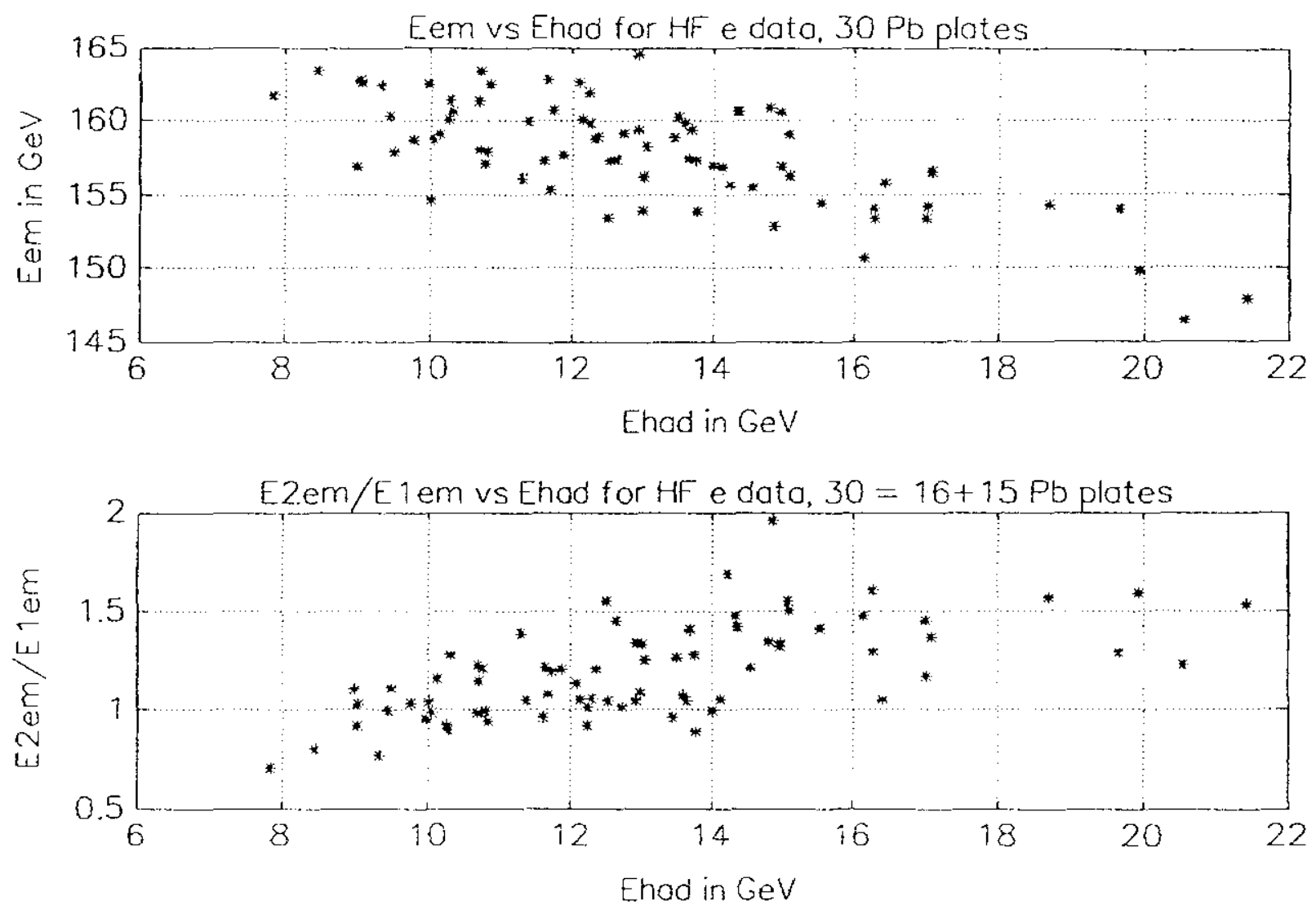

Figure $3 \mathrm{HF}$ data for $170 \mathrm{GeV}$ incident electrons with the EM stack truncated to a depth of 30 plates of $1 / 8 " \mathrm{~Pb}$.

a. Energy in the truncated EM calorimeter compartment vs the remaining energy in the rest of the HF stack (defined to be Ehad).

b. Energy in the truncated EM calorimeter compartment with longitudinal segmentation. Energy ratio E2em/E1em vs Ehad. 


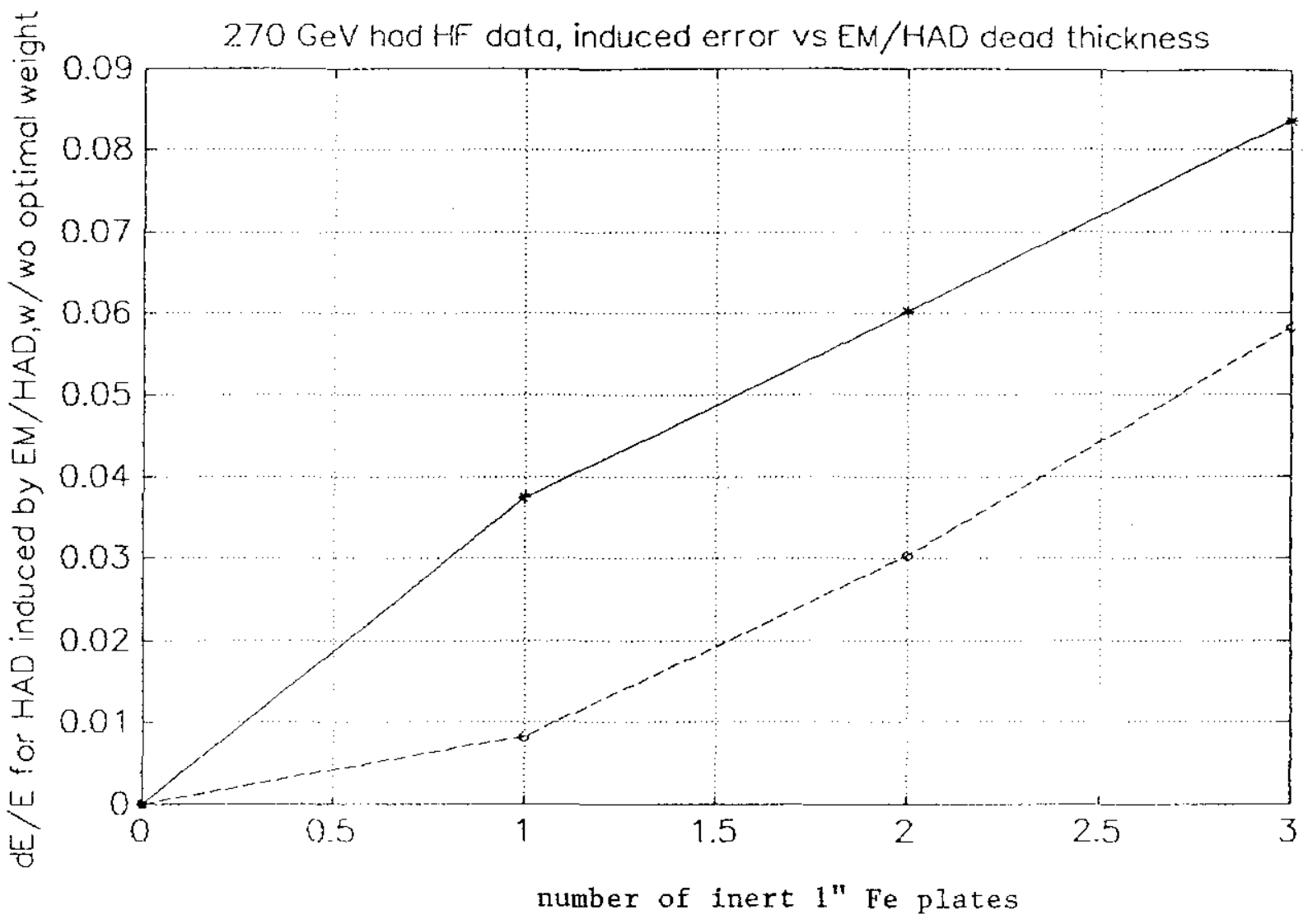

Figure $4 \mathrm{HF}$ data for $270 \mathrm{GeV}$ incident pions. Fractional energy error due to the existence of a number of inert 1 "Fe plates at the EM/HAD boundary. The solid line is without corrections; the dashed line is the result corrected by "optimal weighting" of the first tile downstream of the inert plates. 


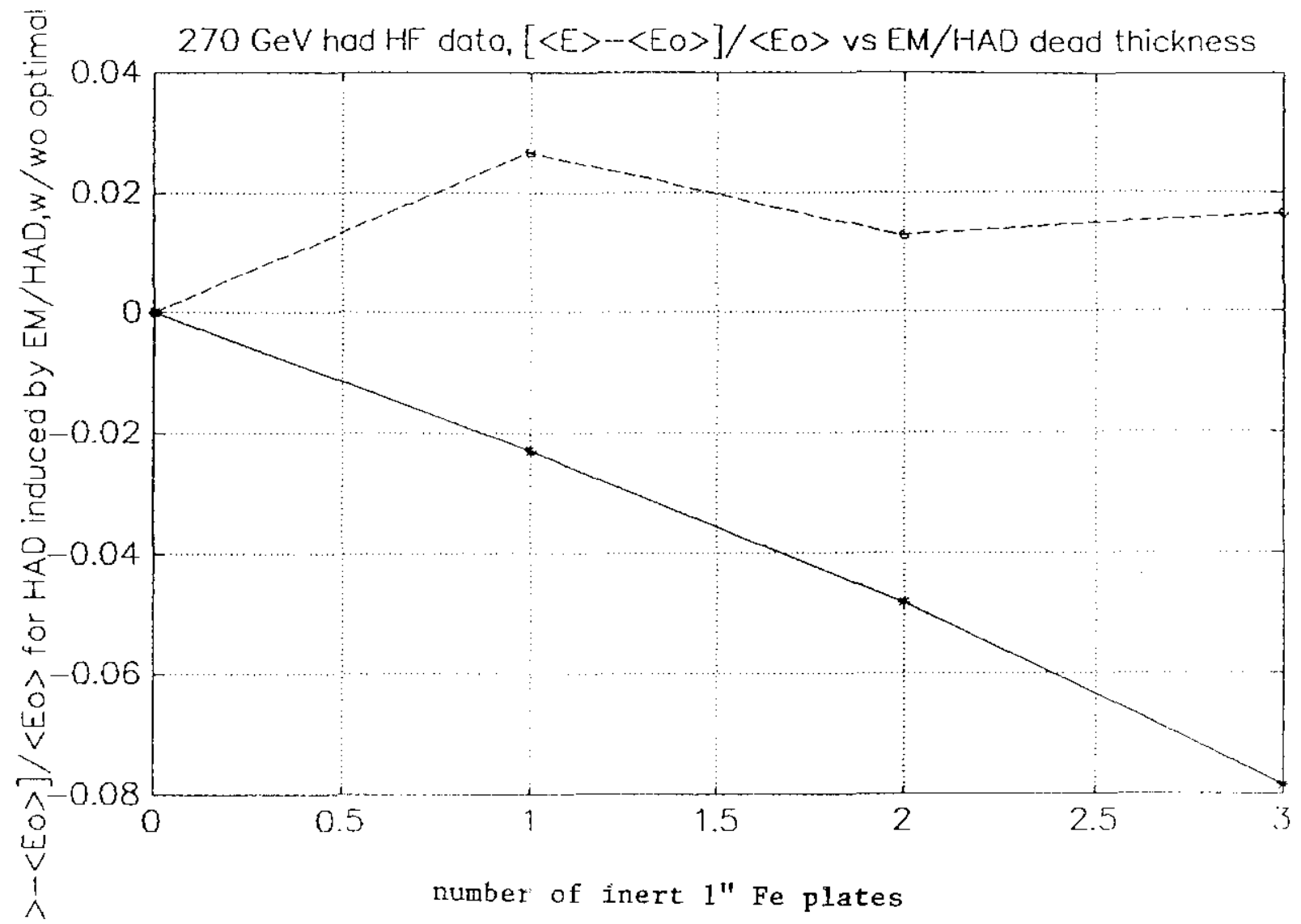

Figure $5 \mathrm{HF}$ data for $270 \mathrm{GeV}$ incident pions. Fractional shift in the mean energy induced by the existence of a number of inert 1" Fe plates in the EM/HAD boundary. The solid line is without corrections; the dashed line is the value corrected by "optimal weighting" of the first tile downstream of the inert plates. 


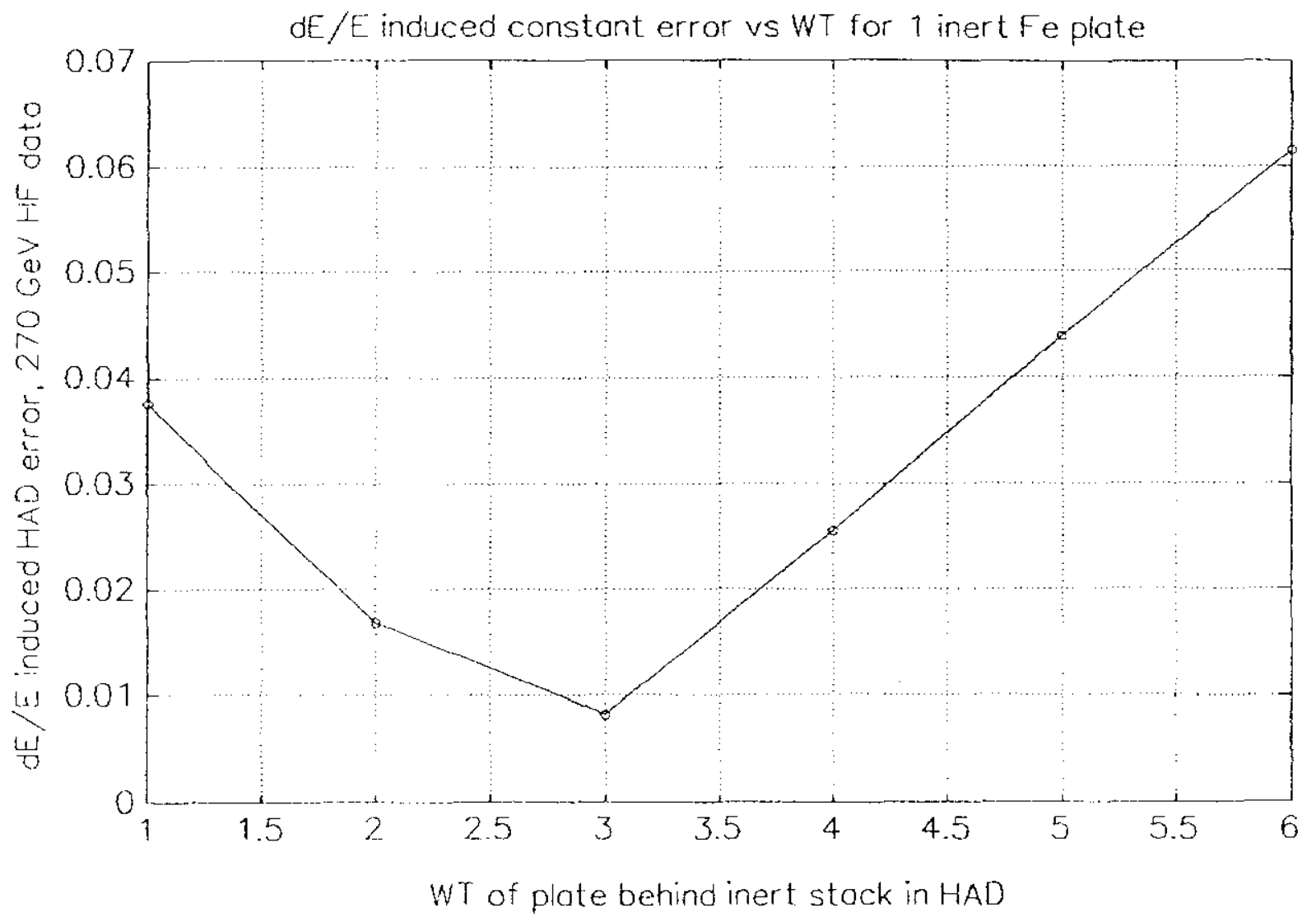

Figure $6 \mathrm{HF}$ data for $270 \mathrm{GeV}$ incident pions. The fractional energy error induced by the existence of one 1" Fe plate is plotted as a function of the light output "weight" of the tile immediately behind the inert plate. The value at the minimum is plotted in Fig. 4 as the dashed line of "optimal weighting". 